\title{
A CLINICAL AND EXPERIMENTAL STUDY OF THE EFFECTS OF EXERCISE ON MOTOR WEAKNESS IN NEUROLOGICAL DISEASE
}

\author{
BY \\ J. A. R. LENMAN* \\ From the Neurological Unit, Northern General Hospital, Edinburgh
}

\begin{abstract}
Muscular weakness is a common neurological symptom which may result from a primary muscular disorder but is more frequently due to interference with the innervation of the muscles. Often there is no specific therapy for the disease responsible and it is necessary to decide on appropriate treatment for the affected muscles, in particular whether they should be rested or given exercise. If exercise is indicated it is important to know the most effective method of carrying it out.

The effect of a programme of exercise given to a group of neurological patients was studied by making quantitative measurements of muscle strength. The same routine of exercise was also applied to a group of healthy volunteers. A smaller group of patients carried out additional exercises designed to produce severe fatigue and the effects of this were measured.
\end{abstract}

\section{Material and Methods}

The biceps and triceps of the upper limb were selected for study as their strength can be measured comparatively easily. Patients with weakness of either of these muscles were studied if they were available for sufficient time and could cooperate in the experiments. All patients admitted over a period of two years to the Neurological Unit at the Northern General Hospital who satisfied these conditions were included.

The measurements of muscle strength were made with a dynamometer designed to register the isometric tension of the biceps or triceps. It consists of a duralumin lever which rises from a spindle on a wooden board. The patient lay with the upper arm resting on the board and the elbow flexed to $90^{\circ}$ so that he was able either to push or pull with the wrist against a handle at the upper end of the lever. This movement was resisted by a spring and registered by a pointer on a scale. At the beginning of each experiment the scale was calibrated in pounds with a spring balance. A tension of $30 \mathrm{lb}$. corresponded with a movement of the wrist of about $3 \mathrm{~cm}$.

For certain purposes a modified version was used. A similar lever with a handle deflected a spring steel bar

* Present address: Neurological Unit, Royal Victoria Infirmary, Newcastle upon Tyne. to which it was attached at its lower end, the steel bar replacing the spring as the load. Resistance strain gauges cemented to the spring steel bar formed two sides of a Wheatstone bridge circuit fed by an A.C. voltage and kept in balance when no force was applied to the lever. The out-of-balance voltage, which is directly proportional to the deflecting force, was amplified and recorded by a pen recorder, so that a permanent record of muscle tension was obtained. It was calibrated in pounds with a spring balance and very powerful pulls produced a movement of the handle of only a few millimetres.

This apparatus was used to make measurements of muscular strength and to carry out training sessions of isometric exercise. A training session consisted of $\mathbf{3 0}$ maximal isometric contractions carried out at 30-second or one-minute intervals (after Darcus, 1956). The strength of the subject each day was taken as the mean of the first five contractions carried out. The percentage alteration in strength was calculated from the difference between the means of the first five observations made in each of the first three and each of the last three training sessions and also from the regression for the means of the first five observations of each of the training sessions. Measurements with the two dynamometers are closely comparable but in general the spring dynamometer was used except where a permanent written record of a contraction was necessary.

Simultaneous measurements were made of the isometric tension of the biceps or triceps and the integrated action potentials obtained from surface electrodes. The electrodes were silver discs of $1 \mathrm{~cm}$. diameter placed $3 \mathrm{~cm}$. apart on the belly of the muscle. The action potentials were amplified and fed into an integrator circuit which first made them unipolar and then applied them to a circuit which gave off pulses at a rate proportional to the mean voltage of the electromyogram (Bates and Cooper, 1954). These pulses were recorded by a pen recorder alongside a simultaneous record of the isometric tension. The electromyogram was calibrated in millivolts by passing sine waves of known voltage through the system.

A small group of patients was given weight lifting exercises. A heavy weight was lifted from waist to shoulder level repeatedly till the subject could no longer raise it. After a short rest the procedure would continue. The sessions lasted up to an hour and were designed to investigate the effects of fatigue. 


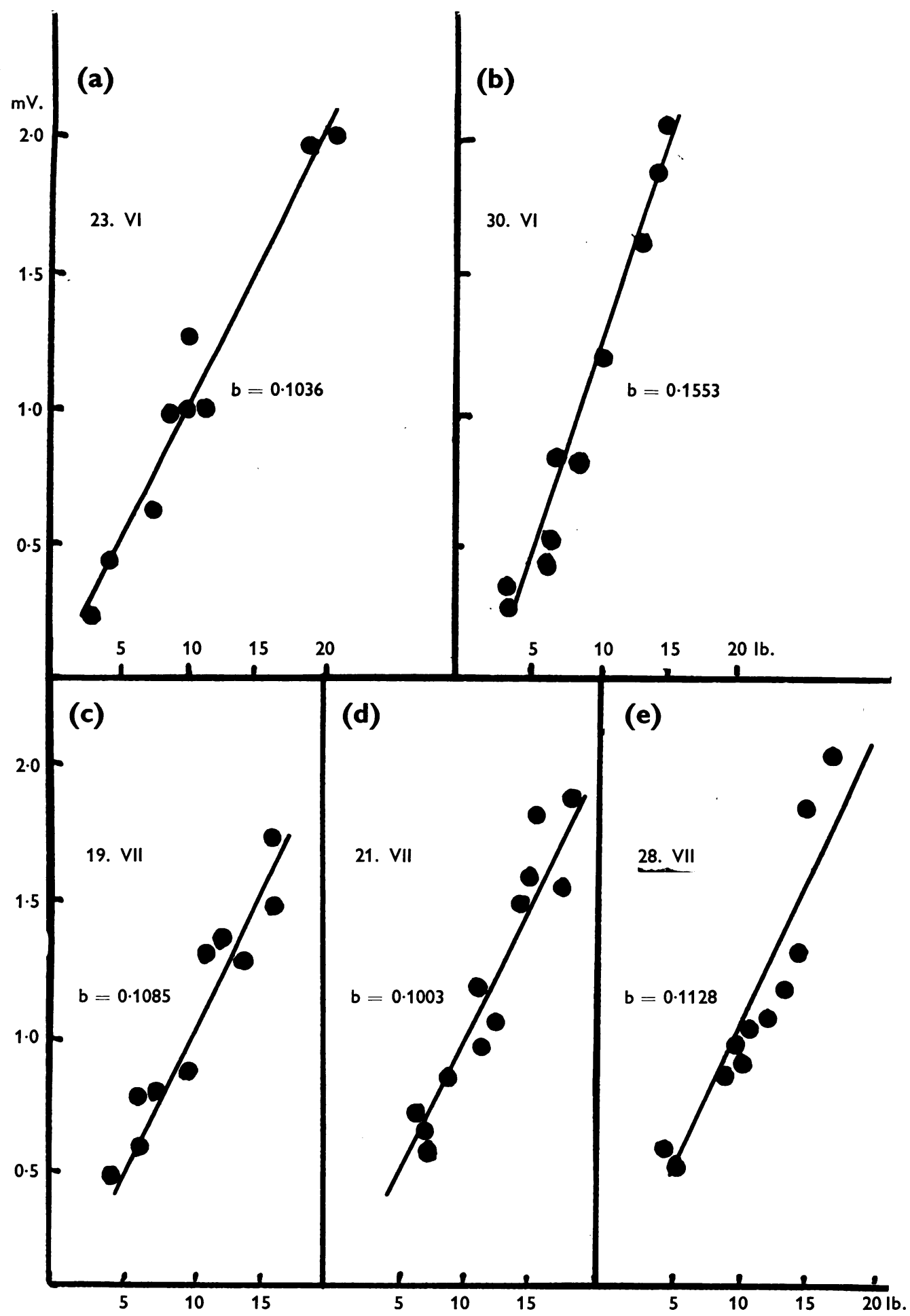

FIG. 1.-Relationship between voltage and tension of left triceps of Subject M.F.L. on five different occasions. For curve (a) regression equation is $Y=0.1036 \mathrm{X}-\mathbf{0 . 0 2 0 0}$. Slope of curve is represented by regression coefficient $\mathrm{b}$ for which the $95 \%$ confidence limits are \pm 0.01606 giving a range of 0.0876 to 0.1169 . The regression coefficients for all the other curves except curve (b) lie within these limits. The correlation coefficient of curve (a) with a straight line is 0.9849 . 


\section{Results}

Relationship between Tension and Integrated Electromyogram.-Simultaneous records during graded contractions were made of the isometric tension and the integrated action potentials of the biceps or triceps muscles of all the healthy volunteers and 14 of the patients in this series. The tension (lb.) was plotted against the mean voltage of the E.M.G. (mV.).

In each instance an increase in tension corresponded with an increase in voltage recorded from the muscle. The relationship between the two variables showed a high degree of correlation with a straight line (Fig. 1). The slope of the curve relating tension to mean voltage varied according to the conditions of the experiment, in particular the position of the electrodes and their distance apart. It also depended on the state of the muscle, and the observation of Edwards and Lippold (1956), that the slope of the curve alters when the muscle is fatigued, was confirmed repeatedly.

The relationship between voltage and tension of a muscle was determined on different occasions separated by intervals of up to several weeks. Care was taken to place the electrodes on the same position on the muscle (marking the site between experiments with adhesive tape) and to reproduce the experimental conditions as closely as possible. Fig. 1 shows the result of one such experiment. The slopes of the regression for each curve are similar. Fig. 2 shows the effect of a trick movement in a subject who would use his shoulder muscles to assist his triceps in a maximal contraction.

Exercise Experiments on Healthy Individuals.The range of normal values for the present technique was determined in six normal subjects using the isometric training sessions described above (Table 1).

Subjects L.F. and M.N. had approximately six training sessions per week whereas the others had exercise three or four times weekly. In each case there was a highly significant progressive improvement in the maximum tension developed, which became evident during the first nine sessions of exercise. Three of the subjects continued for 25 sessions, at the end of which improvement was still taking place (Fig. 3).

Disease of Muscle.-Six patients with weakness attributable to muscular disease were studied. The results of isometric exercise in these patients are summarized in Table $\mathbf{I}$ but it is necessary to consider the individual cases in greater detail.

Case 1 (MN 1667).-A boy, aged 16, with facioscapulo-humeral muscular dystrophy carried out 75 sessions of isometric exercise with his left biceps (Fig. 4).

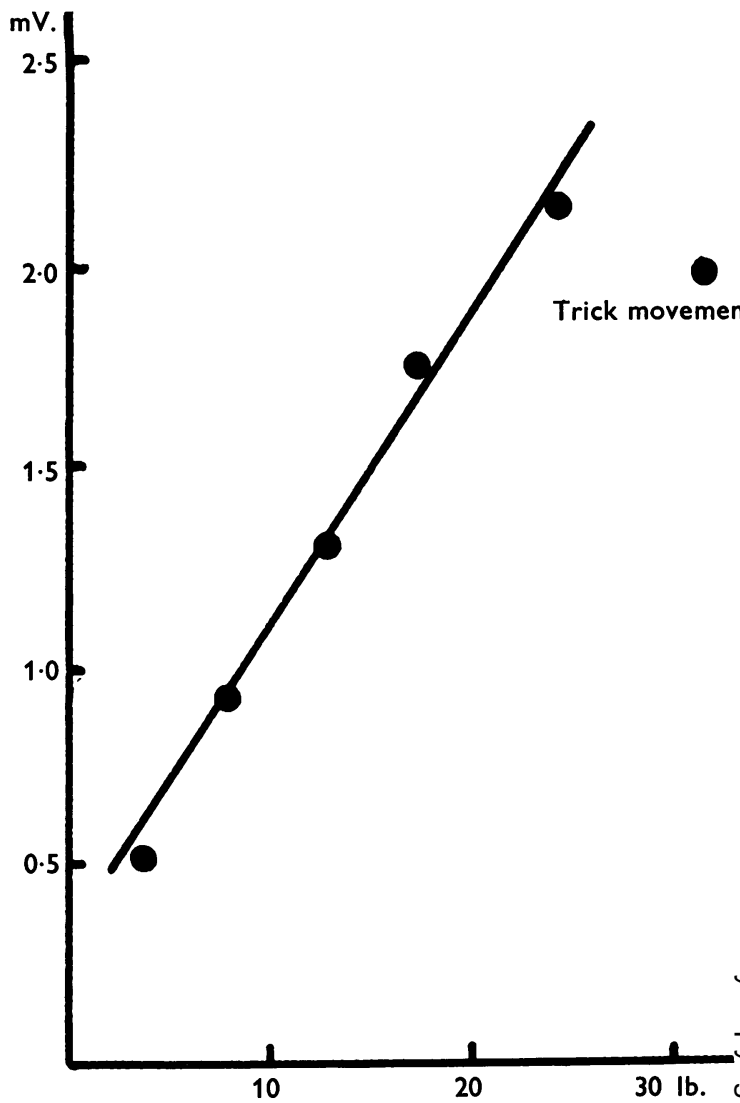

FIG. 2.-Voltage tension curve from Case 23 showing trick movement assisting left triceps muscle. A thrust of $30 \mathrm{lb}$. is associated with the same voltage from the triceps muscle as one of $24 \mathrm{lb}$. because the additional force is derived from the shoulder muscles.

After 10 sessions the muscle was less readily fatigued as there was a smaller fall in tension at the end of each session. After 19 sessions there was an increase in maximal voluntary tension which became progressively larger during the next 30 sessions. During the last 25 sessions the strength of the muscle declined. In the last fortnight of training he carried out weight-lifting sessions which produced severe fatigue and made the muscles sore for several days. During this period there was-no significant change in the rate of deterioration.

The right biceps was weaker than the left. Regular isometric measurements of the strength of the arm (mean of five pulls) were made and during the first month there was no change. A weight-lifting programme was then started and 49 sessions were carried out. Strength increased during the first 25 sessions but after that there was little change. These exercises produced considerable fatigue but were less strenuous than the weight-lifting exercises carried out by the left arm.

Measurements of strength with this patient were made difficult by the fact that the brachio-radialis muscles were powerful and assisted the weak biceps to flex the 
TABLE I

EFFECTS OF ISOMETRIC EXERCISE

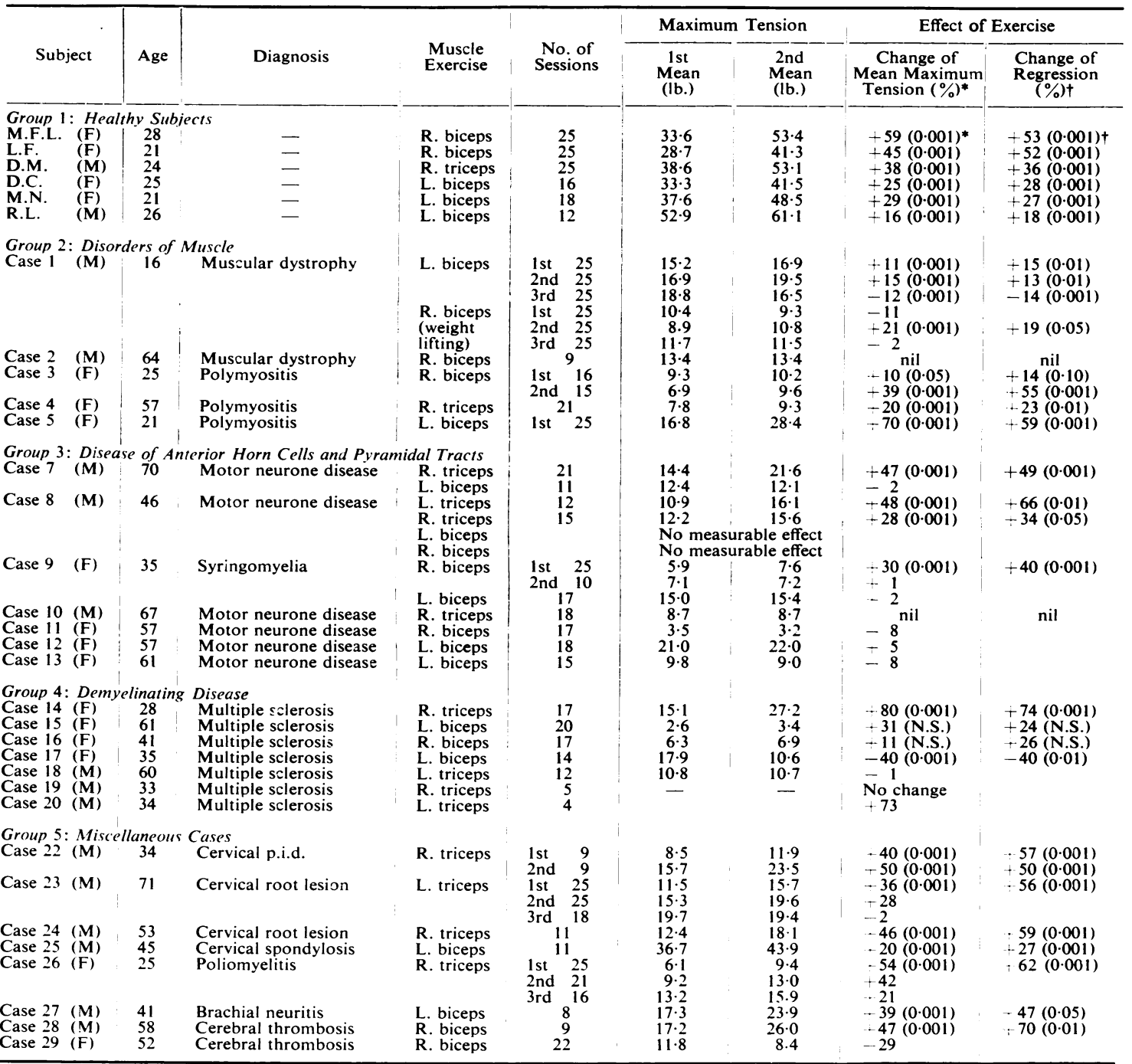

elbow. Repeated girth measurements of the upper arm, however, showed his increase in strength to be accompanied by an increase in girth around the biceps and his deterioration by a decrease in girth.

Twenty-one months later the left biceps was one third as strong as initially. The right biceps became only slightly weaker than when he was first seen.

Case 2 (MN 1934).-A man, aged 64, with facioscapulo-humeral muscular dystrophy carried out nine sessions of isometric exercise with the right biceps with no effect.
Case 3 (MN 1259). - A woman, aged 25, had polymyositis confirmed by biopsy and electromyography. She was under observation for three years and had an episode of respiratory failure when she required intermittent positive pressure respiration.

Her courses of isometric exercise for the right biceps were separated by nine months. The first was of 16 sessions, and the small increase in strength observed was not evident until after the ninth session when she started to take prednisolone. Her strength continued to increase during the three weeks following the exercise when she 


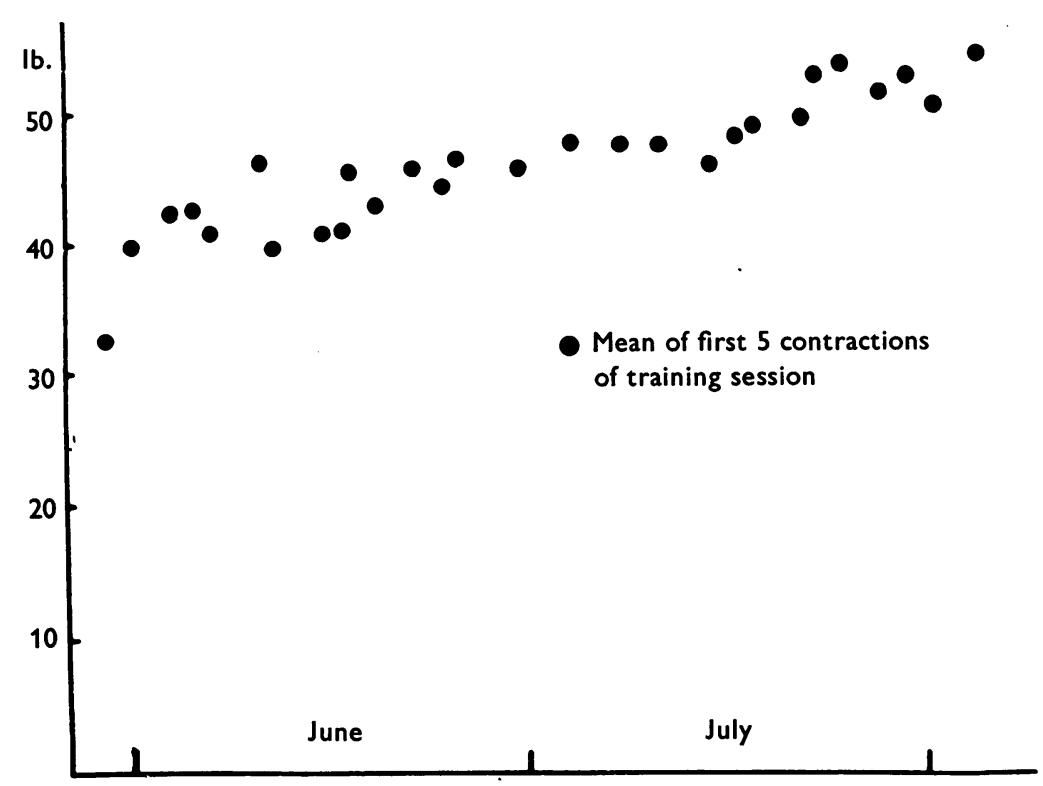

Fig. 3. - The effect of training on the strength of the triceps of a normal subject, D.M.

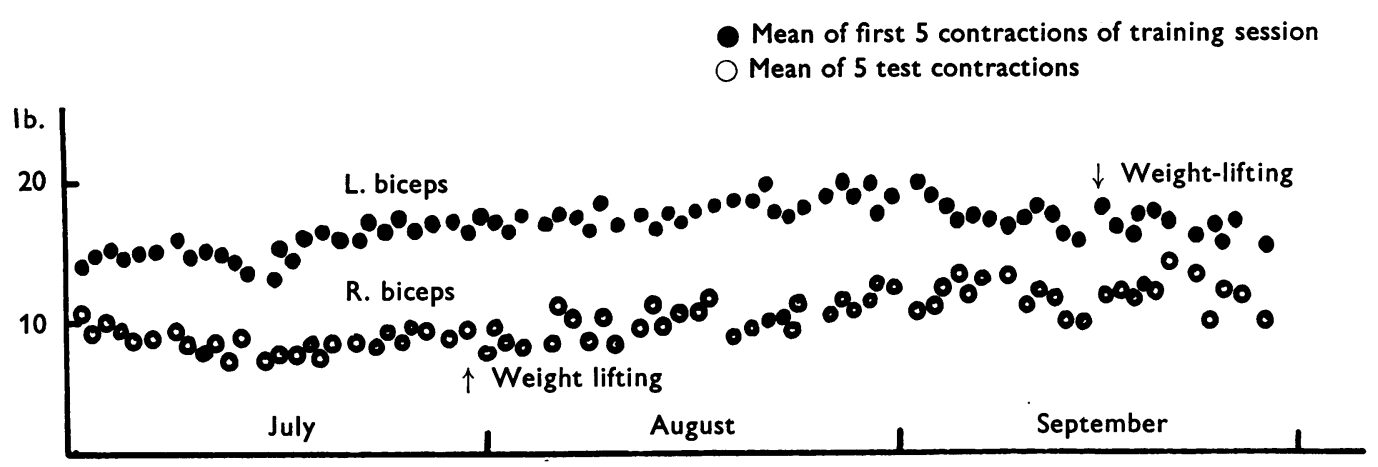

FIG. 4.-The effect of training on Case 1 (muscular dystrophy).

was still taking prednisolone. Her second course of 15 sessions was during recovery from relapse following influenza, when she was again having steroid therapy. A marked improvement occurred in the limb exercised. The other arm improved more slowly but six weeks later there was no difference in strength between the two biceps muscles.

Two things had an adverse effect on her general condition and muscle power. Her three most serious relapses were following febrile illnesses, successively glandular fever, pyelitis, and influenza. Secondly any circumstance that confined her to bed resulted in a loss of strength which would recover rapidly with physiotherapy.

Case 4 (MN 2189).-A woman, aged 57, had weakness of the shoulder girdle and upper arms, as a result of polymyositis. The strength of the right triceps was slightly increased during 21 sessions of isometric exercise. It was not evident until after the tenth session when she started to take prednisolone. After discharge from hospital there was a steady deterioration in strength and general condition and she died nine months later.

Case 5 (MN 1714).-A woman, aged 21, had a proximal weakness of all four limbs which had developed gradually over three and a half years. Her condition ran a fluctuating course and a diagnosis of polymyositis was made although histological proof was not obtained.

She carried out 70 sessions of isometric exercise with the left biceps over five months. Measurements were made of the strength of the right biceps and both triceps muscles. The striking change in the strength of the left biceps corresponded closely with improvement in those muscles which were not exercised (Fig. 5). Although 


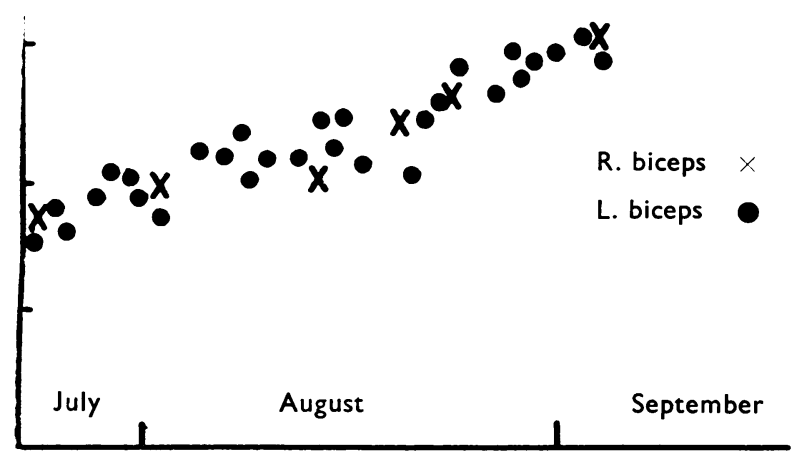

Fig. 5.-The effect of training on the left biceps of Case 5 (polymyositis) showing the corresponding changes in the contralateral muscle.

improvement began before she started steroid therapy, it reached its peak during a course of prednisolone given at this time.

When carrying out a training session her strength tended to fall off towards the end of the session. She also showed a delay in the build up of a contraction so that on a maximal voluntary effort she would take 5 to $7 \mathrm{sec}$. to attain a maximal tension compared with the normal figure of 0.5 to $1.0 \mathrm{sec}$. This has not been observed in other patients with polymyositis or with myasthenia gravis (Simpson and Lenman, 1959).

Case 6 (MN 3119).-A man, aged 50 , had dystrophia myotonica and his arms had begun to weaken. On six days he carried out weight-lifting sessions with the left arm strenuous enough to cause prolonged fatigue. The longest of these lasted one hour and five minutes and in it he lifted 7 lb. from waist to shoulder level 339 times. The strength of the biceps was measured repeatedly and during the five weeks he was in hospital no change was noted.

Five cases of muscular disease showed an increase in strength with exercise but in four it could have been due to other treatment or to spontaneous recovery. The improvement in Case 1 was apparently a true training effect, but it was slower than that seen in normal subjects and it 4 Case 11. was vitiated by the subsequent course of the disease. No adverse effects from fatigue were noted, but, on the contrary, with Case 3 any period of enforced rest in bed was followed by a deterioration.

Degenerative Disease of Anterior Horn Cells and Pyramidal Tracts. - This group includes six cases of motor neurone disease and one of syringomyelia, and the results of isometric exercise are summarized in Table 1.

Case 7 (MN 2103).-A man, aged 70, had weakness of all four limbs due to motor neurone disease. During a course of 20 sessions of isometric exercise the strength of the right triceps increased by $45 \%$ (Fig. 6a). Three months later all his muscles were weaker and no change in strength was recorded during 11 sessions of isometric exercise with the left biceps (Fig. 6b). He next performed weight-lifting exercises with the left arm which were made progressively more strenuous until he was carrying out several times daily a session severe enough to cause prolonged aching of the arm. Isometric measurements made repeatedly on the biceps showed no change in strength. After leaving hospital his condition gradually deteriorated and he died three months later.

Case $8(M N 339)$.- A man, aged 46, had a 10 -year history but the clinical picture was that of motor neurone

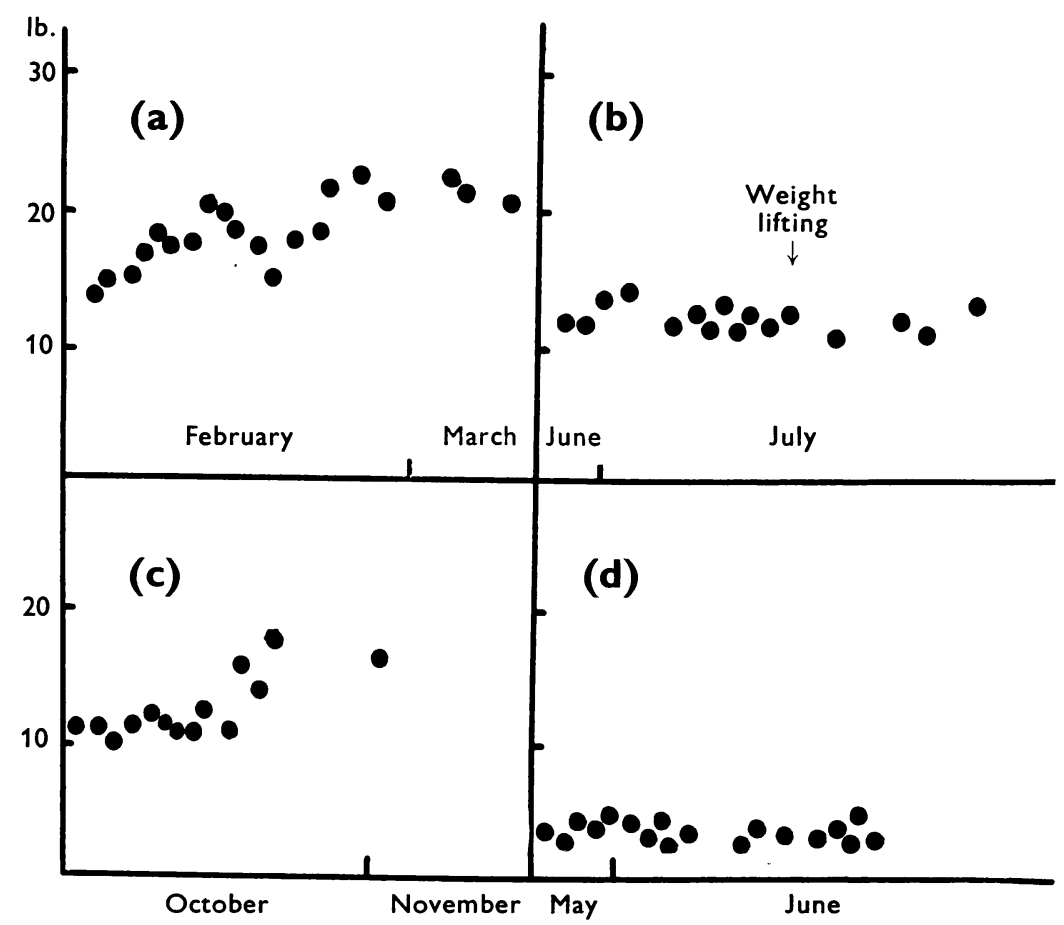

FIG. 6.-The effect of training on four muscles in patients with motor neurone disease. (a) Right triceps of Case 7. (b) Left biceps of Case 7. (c) Left triceps of Case 8. (d) Right triceps of 
disease. He had a pseudo bulbar palsy with weakness of all four limbs, marked wasting of the hands and arms, and widespread fasciculation.

He carried out a series of isometric sessions, each of 30 contractions, at one-minute intervals first with the left (Fig. 6c) and then with the right triceps. In each case there was an increase in strength which was of value as it enabled him to push himself from the lying to the sitting posture and so rise from his bed unaided. He found the exercise sessions fatiguing and after them his arm would ache for 30 to 45 minutes. During a session the maximum tension would fall by $25 \%$. Soon after he started the exercises he noticed fasciculation in the triceps. This fasciculation, which was particularly coarse, involving the contraction of quite large bundles of fibres, was most marked at the end of an exercise session.

The biceps muscles were so weak that he found it difficult to flex his arms against gravity. Each biceps was given a course of isometric exercises but no improvement occurred.

Rest in bed affected him adversely. This he often noticed when he had a cold and on one occasion it was necessary to admit him to hospital for physiotherapy after two weeks in bed following an appendicectomy.

Case 9 ( $M N$ 1068).-A woman, aged 37, who had weak arms as a result of syringomyelia attended two to three times weekly over several months. She carried out 35 sessions of isometric exercise with the right biceps and an increase in strength of about $30 \%$ was recorded, most of it quite early. The exercises produced little fatigue and frequently at the end of a session she would record stronger contractions than at the start. At the end of her fifth session she was reaching tensions equal to those she attained at the start of her twenty-first session. She next carried out seven sessions of weight-lifting exercise over a month. These produced a sensation of fatigue in the arm but there was no further increase in strength. Subsequently strength reverted to its original level.

Her left arm was stronger than the right and a course of 17 sessions of isometric exercise carried out with the left biceps was without effect.

The remaining four patients in this group, Case 10 (MN 2333), Case 11 (MN 2338) (Fig. 6d), Case 12 (MN 2801), and Case 13, had motor neurone disease. They each carried out isometric exercise with a weak upper limb muscle with no effect. Case 13 (MN 3229), a woman, aged 61 , in addition carried out six $\bar{Z}$ sessions of weight-lifting exercises with the right $\stackrel{\mathbb{D}}{\leftarrow}$ biceps during eight days. These resulted in pro- 음 longed aching of the muscle but had no effect on her strength.

All the cases in this group were right handed. जo Cases 7, 12, and 13 were affected earlier or more $\frac{\rho}{\partial}$ severely on the left side. Cases 8,10 , and 11 were affected more severely on the right side. Case 9 was $\overrightarrow{2}$ worse on the right side at the time she came under study but for 10 years previously the left upper $\stackrel{\vec{S}}{\rightarrow}$ limb had also been affected.

In this group of seven patients 12 muscles were $\underline{\bar{\sigma}}$ exercised systematically. Four of the muscles $\frac{\bar{\omega}}{\sigma}$ exercised became stronger. With Case 8 the iso- $\stackrel{\Phi}{\varrho}$ metric exercises produced moderate fatigue. Cases 7, 9, and 13 carried out weight-lifting exercises designed to produce fatigue. No adverse effects were recorded following this treatment.

Demyelinating Disease.-Of the eight cases studied seven carried out isometric exercises and the results of these are summarized on Table $I$.

Cases 14 (MN 1653) and 20 (MN 2267) showed a N more rapid increase in strength than any observed in healthy individuals, which corresponded with change in other muscles not exercised and with $\stackrel{2}{7}$ 을 clinical improvement. Cases 15, 16 (MN 1950), (MN 2747), 18, and 19 (MN 1064), were at a stage

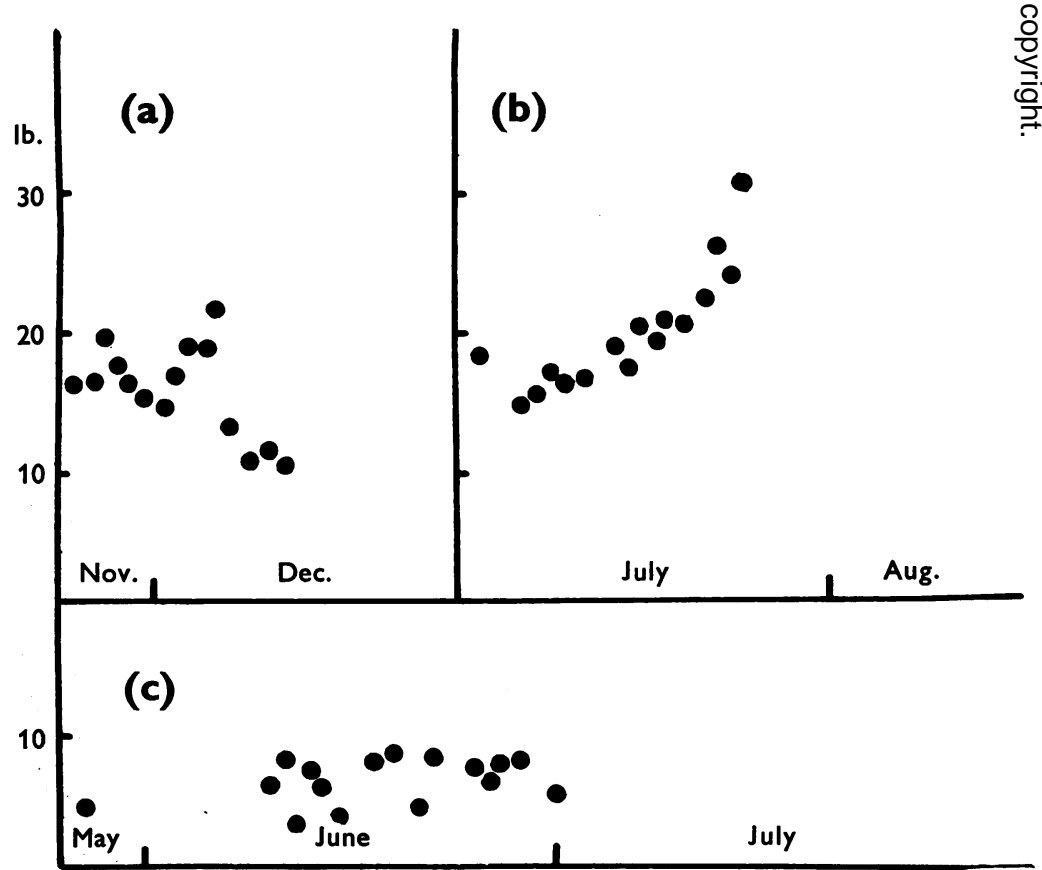

FIG. 7.-The effect of training on three muscles of patients with demyelinating disease. (a) Left biceps of Case 17. (b) Right triceps of Case 14. (c) Right biceps of Case 16.

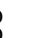


where the disease was fairly static. Case 18 showed no increase in strength with 12 sessions of exercise. Cases 15, 16, 17, and 19 showed a marked variability in strength both from day to day and between the individual contractions of the training sessions (Fig. 7). This made it difficult to discern a consistent trend. Thus Cases 15 and 16 showed a striking apparent improvement but in neither case was the difference between the means or the variance ratio for the regression significant. Case

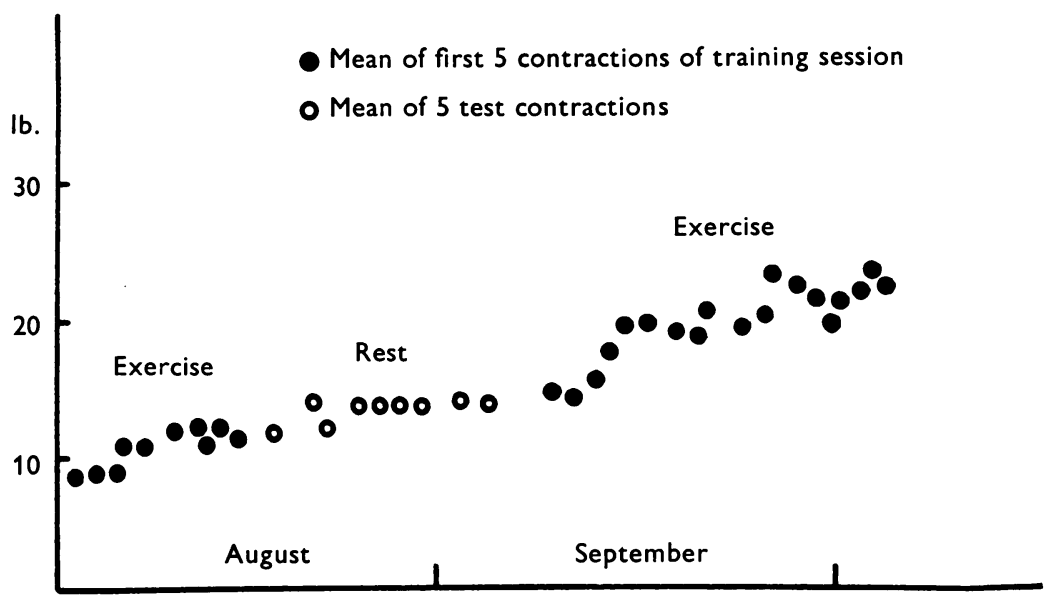
17 showed an apparent deterioration which was significant, but, as Fig 7a indicates, it was not part of a steady trend, and it was not matched by her general condition which was improving.

Only with Cases 15,18 , and 21 was the diagnosis of disseminated sclerosis in any doubt. The alternative with Case 15 was basilar insufficiency and with Case 18 cervical spondylosis. Case 21 had a demyelinating illness but certain features in his case suggested Devic's disease rather than disseminated sclerosis.

Case 15 (MN 125).-A woman, aged 61, found the isometric exercises, of which she carried out 20 sessions, unusually fatiguing. This may account in part for her day-to-day variation in strength for she always did badly when she was tired. It was not evident that fatigue produced permanent ill effects but it would prevent her carrying out the next exercise session adequately.

Case 18 (MN 1722).-A man, aged 60, had weakness of the right arm and both lower limbs. He was admitted twice to the Neurological Unit, each time after a period of rest in bed after a respiratory infection which had been followed by increased stiffness of the lower limbs.

On his first admission 12 sessions of isometric exercise with the right triceps resulted in no increase in strength. Six months later the strength of the right biceps and triceps was found to have improved spontaneously although the lower limbs had grown weaker. He was given a course of weight-lifting exercises for the right biceps in which he carried out sessions on 16 days which produced stiffness of the arm muscles and marked generalized fatigue. Repeated measurements of the biceps and triceps muscles showed no change and his general condition improved.

Case 21 (MN 3254).-A man, aged 31, was improving after an episode of weakness in the limbs, ataxia, and retrobulbar neuritis eight months previously. When

first seen he had some weakness of the right arm muscles. He carried out nine weight-lifting sessions with the right biceps during two weeks which were made progressively more strenuous, and isometric strength measurements were made. His arm became stronger and the exercises were abandoned when it was found that an hour of heavy weight lifting no longer produced marked Patigue.

The cases in this group which showed a significant increase in strength with exercise were passing into a stage of remission. Case 18 showed a deterioration with rest in bed which was partly reversed with exercise. It was not possible to demonstrate adverse effects from deliberately produced fatigue.

Miscellaneous Conditions. - The eight cases included in this group are summarized in Table $\mathbf{I}$.

Each patient, with the exception of Case 29, showed a significant increase in power in the muscle exercised and in each case this was materially useful to the subject. The treatment of Case 29 (MN 3128), who had a pseudo bulbar palsy and tetraparesis of gradual onset and probably a vascular pathology, coincided with a general deterioration in the patient's condition.

The patients with cervical spondylosis had unilateral weakness so it was not possible to compare one side with the other. In addition to exercise they were treated with neck traction and immobilization 'of the neck in a collar. Case 22 (MN 1736), however, had two courses of isometric exercise for the right triceps which were separated by a rest period lasting 25 days. His strength increased more rapidly when he was having exercise than during the period of rest (Fig. 8). Case 23 (MN 1567) had more than 75 exercise sessions for the left triceps and strength improved by $64 \%$. Case 24 (MN 2001) registered 


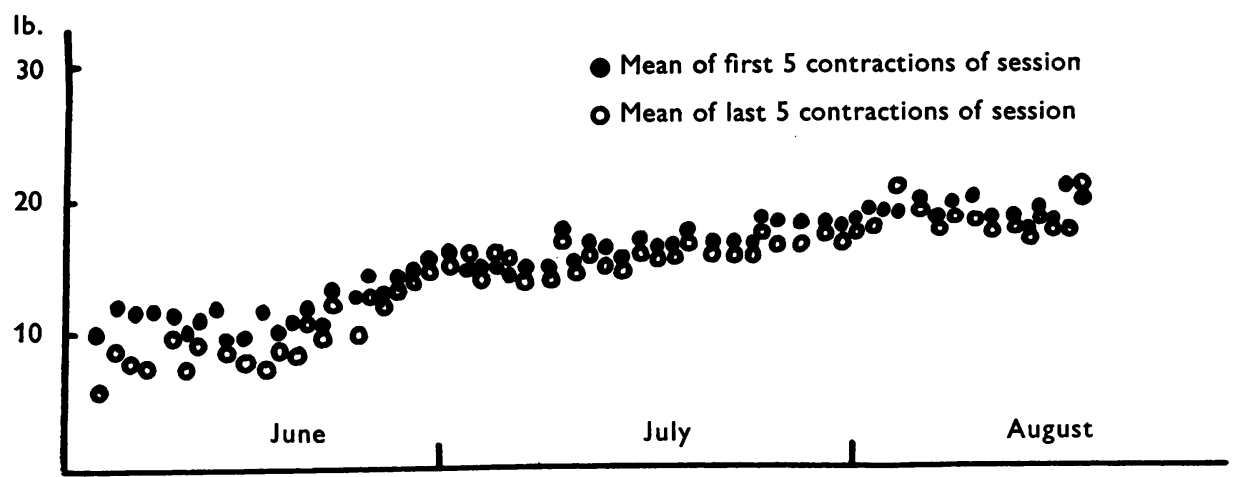

Fig. 9.-Effect of training on left triceps of Case 23 (cervical spondylosis).

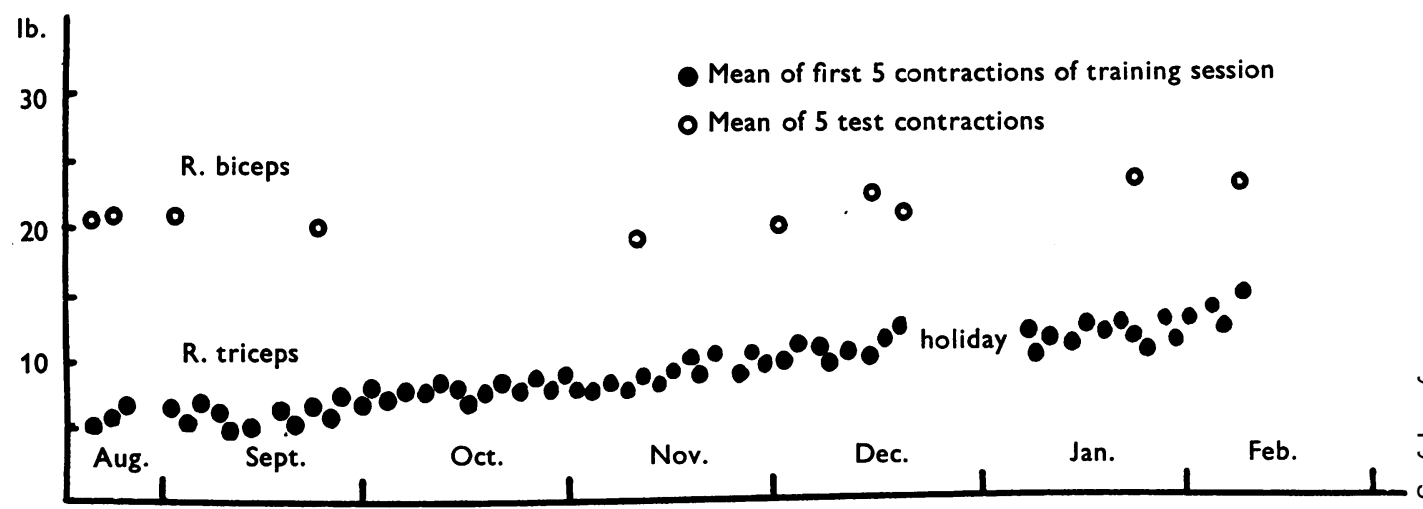

FIG. 10.-Effect of training on right triceps of Case 26 (poliomyelitis).

an improvement of $46 \%$ during 11 sessions of exercise with the right triceps. With Case 23 there was no improvement in maximum voluntary tension until after 12 sessions of exercise but before that there was a fall in the percentage decrement per session (Fig. 9). After 50 sessions strength was not further increased but he learned to increase his thrust against the dynamometer by a trick movement using his shoulder (Fig. 2). A subsequent intensive course of exercise using sessions of 60 contractions at 30-sec. intervals instead of 30 at one-minute intervals produced no further change.

Case 25 ( $M N$ 2699).-A man, aged 45, had enough radiological evidence of cervical spondylosis to account for pain in the shoulder, weakness of the left upper arm, and sensory symptoms in the lower limbs, but he also had wasting of both first dorsal interossei and widespread fasciculation which suggested that he might be developing motor neurone disease. However, with neck traction and 11 sessions of exercise for his left biceps the strength returned nearly to normal and nine months later this improvement was still maintained.
Case 26 (MN 1340).-A woman, aged 25, had had poliomyelitis eight months before. During 62 sessions of isometric exercise for the right triceps the strength of the muscle increased by $110 \%$. During a pause of 19 days $\Phi$ in this treatment the power of the arm did not change (Fig. 10). The other upper arm muscles were not exercised and improved more slowly. The increase in strength 3 of the triceps continued till she ceased to exercise it. After that there was a slight falling off but it remained at about double its initial strength and nine months later no further change had taken place.

Case 27 (MN 2369).-A man, aged 41, developed a $\dot{0}$ flaccid paresis of the arms with pain in the shoulders and C.5 sensory loss following a respiratory infection. 8 He was treated with prednisolone and physiotherapy and $₹$ his strength improved steadily over several months. After finishing his course of prednisolone he carried out eight sessions of isometric exercise with the left biceps, $\frac{D}{2}$ which appeared to improve more rapidly during the course of exercise than in the period preceding it, al- $N$ though his skill in developing trick movements made him difficult to assess.

Two weeks after admission to hospital he missed two 
days of physiotherapy and an increase in the pain and stiffness of the arms was recorded.

Case 28 ( $M N$ 2339). - A man, aged 58, had a spastic right hemiparesis as the result of a cerebral thrombosis one year before. Nine sessions of isometric exercise for the right biceps resulted in an increase in strength of $47 \%$. His spasticity, as measured by the ease with which clonus was elicited, became more pronounced.

Seven of the eight patients in this group had a condition in which recovery was taking place. In each of these seven cases the muscle which was exercised became stronger.

\section{Discussion}

Although other aspects of motor function, such as coordination, muscle tone, and endurance, are important, the experimental side of this study has been confined to the effects of exercise on the strength of a voluntary contraction. Other effects are less easy to measure and consideration of them has been based on clinical observations.

The physiological changes which exercise produces in nerve and muscle require to be understood if therapy is to be on a sound basis. Unfortunately little is known about them so that the aim here has been to establish a few of the facts on an empirical basis, in so far as this can be done with a small number of patients. Attention has been directed to three questions, namely, the effect on weak muscles of a system of exercise which has been shown to strengthen healthy muscle, the effect of deliberately produced fatigue, and the effect of confinement to bed without exercise.

It is accepted that systematic exercise will increase the strength of healthy muscle, but there is no agreement as to how this comes about and the most efficient way of developing a muscle by exercise is not known. Both isotonic and isometric exercises are effective, and Darcus and Salter (1955) have shown that if programmes of the same intensity are used the results are comparable. De Lorme (1945) has developed a technique of progressive resistance exercise based on experience gained by professional strength athletes. It consists of weightlifting exercises which are made progressively more strenuous. There are many modifications of the technique but in each the subject lifts the maximum weight of which he is capable a fixed number of times each session. Hettinger and Müller (1953) and Müller and Hettinger (1953) suggest that a less strenuous programme is adequate. They state that a single isometric contraction each day will produce an increase in strength and a contraction of twothirds maximum tension is adequate to produce the maximum possible training effect.

An increase in the strength of a muscle is associated with structural changes. A period of intense exercise produces transient swelling and a prolonged period of training leads to hypertrophy. There is no clear correlation, however, between the degree of hypertrophy and the increase in strength, and MacQueen (1954) differentiates between exercise programmes designed to produce primarily an increase in power and those leading principally to hypertrophy. A neuromuscular adaptation leading to more efficient use of the available motor units may be as important as a structural change in the individual fibres. This is supported by the early observation of Scripture, Smith, and Brown (1894) of cross-education which has been frequently repeated by other workers (Hellebrandt, Parrish, and Houtz, 1947; Darcus and Salter, 1955). If a muscle is exercised the contralateral muscle also becomes stronger.

Recently the effects of exercise on muscles weakened through injury or disease have attracted attention. De Lorme and Watkins (1948) have applied the technique of progressive resistance exercise to the treatment of disuse atrophy resulting from bone and joint disorders. Quantitative studies on the effect of exercise on muscle recovery in poliomyelitis were reported as early as 1916 by Lovett and Martin but opinion is still not unanimous as to its value. De Lorme and Watkins (1948) maintain that resistance exercise carried out over a period of years is valuable in that it develops those muscle fibres which retain their nerve supply and this is supported by the observations of Mead (1950) and Darcus (1955). On the other hand, Sharrard (1955) in a detailed study of the natural history of the disease, while he concedes that exercise may increase the rate of recovery, is doubtful whether it affects the ultimate level of recovery.

Russell (1947) and Horstmann (1950) have shown that exercise during the preparalytic phase of poliomyelitis increases the risk of paralysis supervening in the exercised muscles. This observation indicates that fatigue, apart from its local effects on the muscles, may affect the cells of the central nervous system. The effect of fatigue on muscle recovery in the later stages of poliomyelitis has been studied by Russell and Fischer-Williams (1954). They found that deliberately produced fatigue has no adverse effects on muscle recovery in this disease.

In other neurological conditions quantitative information is scanty. After cerebrovascular accidents active exercises are used increasingly in the treatment of paretic muscles and the clinical impression is probably justified that they hasten the recovery of strength as well as prevent contractures. In disseminated sclerosis exercises are often prescribed to strengthen weak muscles, improve coordination, and lessen spasticity, but the variable 
natural course of the disease has made it difficult to assess their value, and McAlpine, Compston, and Lumsden (1955) have stressed the dangers of fatigue. Walton and Nattrass (1954), in their review of the muscular dystrophies, report adverse effects from rest in bed but suggest that fatigue may also be harmful.

In this study training has been mainly with isometric exercises. These are probably as effective as isotonic exercises (Darcus and Salter, 1955) and since they produce little fatigue it has been possible to study the effects of fatigue separately with a series of weight-lifting experiments. Isometric tension was chosen advisedly as the measurement of strength. Isotonic measurements are unsatisfactory as the maximum weight a subject can move can only be determined after several attempts so that when the final test is made the muscle is fatigued. In making the strength measurements, positioning of the patient was found to be critical so that all measurements were made by the same observer. The consistent linearity of the curves relating tension to the integrated electromyogram, which conforms to the earlier observations of Lippold (1952), has been taken as indicating the infrequency of trick movements when care is taken. The fact that the same curve is reproducible in the same subject on different occasions suggests that the dynamometer measurements are reasonably reliable.

Effect of Isometric Exercise.-The effects of isometric exercise on healthy subjects were consistent in that all the subjects showed a substantial increase in strength. The exercises did not produce fatigue so it is evident that a muscle can be made stronger without fatiguing it.

Of the cases with a primary muscular disorder, only Case 1 showed an improvement which could not be partly explained by spontaneous recovery or the effects of other treatment. The improvement was slow but it is significant that a training effect is possible at all with dystrophic muscle. It cannot be said how far the patients with polymyositis would have improved if exercise had not been given. Changes in the contralateral muscles may have been due to cross-education (Scripture et al., 1894). The only occasions, however, where the changes associated with exercise were striking were during improvement after a relapse. Here the correction of disuse atrophy was an important factor, and it is probably true that the main indication at present for exercise in muscular disorders is in the treatment and prevention of wasting due to disuse.

In the group which includes six patients with motor neurone disease the improvement in four of the 12 muscles exercised could not have been the result of spontaneous recovery as in each case the disease was progressing. Of the four muscles three $\stackrel{\mathbb{C}}{=}$ were triceps which is a muscle used to a limited of extent in everyday life. The fourth muscle was the biceps of an arm so weak that it had fallen largely 0 into disuse. Müller and Hettinger (1953) have shown that if the limb of a healthy subject is im- $\frac{\rho}{\square}$. mobilized in plaster its response to training after disuse atrophy has developed is three times as rapid.. as a training effect observed in the same muscles $\overrightarrow{\vec{F}}$ before immobilization. None of these muscles $\stackrel{9}{\rightarrow}$ showed so rapid an improvement but with Case 7 (Fig. 6a) the improvement during the first nine $\frac{\bar{\sigma}}{\omega}$ sessions was more rapid than with any of the $\frac{0}{\vec{D}}$ normal subjects. The two triceps muscles of Case $8 \stackrel{\varnothing}{\varnothing}$ showed no change during the first eight sessions and के then a striking and rapid change took place. With $\vec{\circ}$ the biceps of Case 9, although no change was noted in the first five contractions of a training session $\vec{\omega}$ until several weeks had passed, toward the end of her fifth exercise session she was working up to a contraction nearly as great as the maximum she attained later. Her exercise sessions were compara- N tively infrequent and it is possible that in eac $\mathrm{P}$ session she had to work off the effects of disuse i⿱ $\vec{\sim}$ the intervening period. Thus in this group the sole effect of exercise may have been the correction o wasting due to disuse.

Of the seven patients with demyelinating diseaso who received isometric exercises, only Cases 14 and 20 showed improvement in the muscle exercised. This was more rapid than is usual for a training of effect and occurred when the disease was entering a remission. The variability in strength which these patients showed from day to day and from contraction to contraction is in keeping with the known character of the disease.

The remaining cases with one exception all improved with exercise. They were patients in whom the disease had a natural tendency to recover but in some it appears that exercise increased the rate of recovery. Case 26 had reached a stage where the natural rate of recovery is slow. The exercised muscle improved at a rate faster than the other affected muscles and during a pause in her treatment progress stopped until she started to exercise again. After she had finally ceased to exercise no further improvement occurred. This result in a case of poliomyelitis is in keeping with the experience of De Lorme and Watkins (1948) and Darcus (1955). Case 22 likewise improved more rapidly during two periods of exercise than during the intervening rest period. Case 28 improved during treatment more rapidly than during the previous year since his original vascular accident. Case 23 did not start to become stronger till 12 days after he had started 
to exercise, and although one can ascribe his improvement to neck traction and a cervical collar it is possible that these merely alleviated the root irritation and allowed the muscle to respond to exercise. It is of interest that with this patient after a certain stage no amount of additional exercise would produce any further change, indicating that the ultimate level of recovery is determined by the amount of structural damage that has taken place.

The Effects of Fatigue.-Although fatigue may contribute to the adaptation of a muscle to exercise the isometric experiments indicate that it is not an essential factor in increasing strength. There remains the question whether it may delay muscle recovery. If it increases the risk of paralysis developing in preparalytic poliomyelitis (Russell, 1947) it may also cause deterioration in other conditions where anterior horn cells are degenerating, such as motor neurone disease. McAlpine et al. (1955) have described patients with disseminated sclerosis whose symptoms first appeared after severe exertion, paralysis developing in the muscles exercised. Patients with muscular dystrophy often ask whether fatigue will do their muscles any harm and it is important to be able to give them appropriate advice.

Although the isometric exercises did not fatigue the healthy subjects a number of the patients with weak muscles complained of aching in the muscles during a session and found that they could not exert as much force at the end of a session as at the start. An improvement in the maximum tension exerted at the beginning of a session was often preceded by a decrease in the decrement of strength during the sessions (Fig. 9) indicating that these exercises had an effect on endurance as well as on strength. Of the seven patients who carried out isotonic exercises which caused considerable fatigue, none showed an increase in strength greater than that resulting from isometric exercises.

The weight-lifting exercises were designed to produce fatigue. In no case did these exercises affect the rate of deterioration or result in deterioration where none was occurring. Of this group of cases two had dystrophic muscle (Cases 1 and 6), two had motor neurone disease (Cases 7 and 13), two had demyelinating disease (Cases 18 and 21), and one had syringomyelia (Case 9).

A further case of motor neurone disease (Case 8) found the isometric exercises to the triceps muscles particularly fatiguing and the sessions were followed by increased fasciculation. The muscles, however, became stronger so that the fasciculation does not necessarily indicate an increase in the rate of anterior horn cell destruction. All six cases of motor neurone disease were right handed but only three showed the disease to be more advanced on the right side of the body.

Two cases with demyelinating disease were deliberately exercised to exhaustion without ill effects. A further two found the isometric exercises fatiguing, but did not suffer any permanent harm. They found that when they became tired they were unable to carry out their exercises adequately.

The amount of exertion carried out by these patients was small compared with that undergone by those who have been described as developing muscular weakness through poliomyelitis or disseminated sclerosis after severe exercise such as climbing or cross country running when they were still fit. Nevertheless the weight-lifting exercises were adequate to fatigue appreciably patients weakened through prolonged illness. They were more fatiguing than any likely to be carried out in the course of treatment.

The number of cases of muscular dystrophy is small, and although it has not been possible to demonstrate any deleterious effects from exercise, it is possible that certain patients, particularly those where the disease is more rapidly progressive, may harm muscles through excessive use.

The Effects of Rest.-Rest was not deliberately prescribed for experimental purposes but certain patients had periods of rest in bed enforced through circumstances or intercurrent disease.

Case 3, who had polymyositis, had three relapses following infections and it is likely that the infection itself was an important disturbing factor. Nevertheless she always noticed that any period of rest in bed, as after a cold, would lead to increased weakness which it would take several days of active use of the muscles to relieve. Muscle weakness, which at the time was associated with stiffness, was most severe during the period of prolonged rest in bed when she was in the respirator.

Case 8, who had motor neurone disease, noticed an increase in spasticity if he was confined to bed and disuse aggravated his weakness.

Disseminated sclerosis often relapses after intercurrent infections which appear to reactivate the disease. Case 20 had a severe relapse which necessitated admission to hospital after an attack of influenza. Case 18, on the other hand, was twice admitted to hospital after an intercurrent infection, not because the disease was more active but because rest in bed had led to an increase in muscular stiffness which was subsequently relieved by exercise.

\section{Conclusion}

The place of exercise in the management of neurological disease is an important but a restricted 
one. As a means of increasing the strength of muscles paralysed by degenerative disease it is probably without value. But these patients may develop weakness of relatively unaffected muscles due to disuse, particularly if they are confined to bed for any reason and this weakness is relieved by exercise. Likewise the affected muscles, if they are spastic, will show an increase in stiffness if they are rested. It is worthwhile to give active exercises to patients who are recovering from a paralytic lesion as these may increase the rate of recovery. They need not cause fatigue to be effective, but any fatigue which they incidentally produce is unlikely to do harm.

\section{Summary}

Isometric exercises are an effective means of improving the strength of healthy individuals. They need not cause fatigue to be effective.

It is possible to increase the strength of a patient with a muscular dystrophy, but very slowly, and progress of the disease may undo any benefit achieved.

Weakness resulting from degenerative disease of the nervous system is not affected by exercise, unless the loss of strength is the result of disuse.

When a paralysed muscle is recovering either through specific treatment, or spontaneously, there is evidence that active exercise increases the rate of recovery.

Patients with motor weakness due either to primary muscular disease or to disease of the central nervous system may deteriorate with rest in bed. This is due in part to disuse, in part to an increase in stiffness. Both these effects may be corrected by active exercise.
No adverse effects have been shown to result from fatigue in primary muscular or neurogenic paresis.

I wish to thank the physicians in the Neurological Unit at the Northern General Hospital, Edinburgh, for permission to study their cases.

Dr. John Marshall originally provided facilities for this investigation. Dr. J. A. Simpson continued to provide them and has been a constant source of encouragement and helpful criticism. Professor A. E. Ritchie has given valuable advice. The cooperation of Miss $\mathbf{S}$. Cameron and her staff in the Physiotherapy Department has been most valuable.

The project was supported by a grant from the Scottish Hospitals Endowments Research Trust which is gratefully acknowledged.

\section{REFERENCES}

Bates, J. A. V., and Cooper, J. D. (1954). J. Physiol. (Lond.), 123, 28P.

Darcus, H. D. (1955). Poliomyelitis: Papers and Discussions presented at the Third International Poliomyelitis Conference [Rome, 1954], p. 446. J. B. Lippincott, Philadelphia. (1956). Personal communication.

and Salter, N. (1955). J. Physiol. (Lond.), 129, 325

De Lorme, T. L. (1945). J. Bone Jt Surg., 27, 645.

-and Watkins, A. L. (1948). Arch. phys. Med., 29, 263

Edwards, R. G., and Lippold, O. C. J. (1956). J. Physiol. (Lond.), 132,677 .

Hellebrandt, F. A., Parrish, A. M., and Houtz, S. J. (1947). Arch phys. med., $28,76$.

Hettinger, T. and Müller, E. A. (1953). Arbeitsphysiologie, 15, 111 . (D)
Horstmann, D. M. (1950). J. Amer. med. Ass., 142, 236.

Lippold, O. C. J. (1952). J. Physiol. (Lond.), 117, 492.

Lovett, R. W., and Martin, E. G. (1916). J. Amer. med. Ass., 66 729.

McAlpine, D. Compston, N. D., and Lumsden, C. E. (1955), Mu tiple Sclerosis, p. 39. E. and S. Livingstone, Edinburgh.

MacQueen, I. J. (1954). Brit. med. J., $2,1193$.

Mead, S. (1950). J. Amer. med. Ass., 144, 458.

Müller, E. A., and Hettinger, T. (1953). Arbeitsphysiologie, 15, 223

Russell, W. R. (1947). Brit. med. J., 2, 1023.

and Fischer-Williams, M. (1954). Lancet, 1, 330.

Sharrard, W. J. W. (1955). J. Bone Jt Surg., 37B, 63.

Scripture, E. W. Smith, T. L and Brown, E. M. (i894). Stud. Yale psychol. Lab., 2, 114.

Simpson, J. A., and Lenman, J. A. R. (1959). Electroenceph. clin. Neurophysiol, 11, 604.

Walton, J. N., and Nattrass, F. J. (1954). Brain, 77, 169. 